\title{
Back to basics on quantum theory
}

\section{Fashion seems to have come full circle as physicists look to the 1920s for inspiration. But what has prompted the change?}

WHY is there, just now, so much interest in the foundations of quantum mechanics? The simplest explanation would be that the grand old men of quantum mechanics the young men of the mid-1920s - are all now dead, so that the field is clear for a reexamination of the subject by a succeeding generation. But this is not the case. P.A.M. Dirac, who ranks with the late Wolfgang Pauli as one of those who made quantum mechanics into a coherent whole in the second half of the $1920 \mathrm{~s}$, is fortunately still alive and active, commuting with the seasons between Cambridge (England) and Tallahassee (Florida).

There are several candidate explanations, of which the most obvious is extrinsic to quantum mechanics as such: half a century after the ending of the most radical revision of the notion of physical reality that began with Planck (1901) and ended with the recognition that the matrix mechanics of Born, Heisenberg and Jordan (1925) and the wave mechanics of Schrodinger (1926) were different ways of saying the same sort of things, people have come naturally to a reconsideration of what really occurred during that upheaval.

The more probable explanation is practical - people's need to tell a more sophisticated generation of students a coherent tale about a field of physics which is acknowledged to be the most searching account of what the physical world is like but which has been uncomfortably balkanized almost from the beginning, and which is now not one subject but half a dozen. At one extreme there is what passes as quantum mechanics among chemists. On the convenient assumption that the nuclei of atoms in a molecule are fixed at their mean positions (for which the BornOppenheimer approximation provides the justification), people have become extremely skilled at calculating electronic energy levels. Another sub-branch of wave mechanics is that in which similar techniques are carried over into the treatment of the properties of nuclei. At the other extreme is that other, more esoteric, pursuit generally labelled as the theory of quantum fields from which have sprung two great successes, the successful treatment of quantum electrodynamics and the unification of the fields of electrodynamics and the weak nuclear interaction, but which seems to be running into the ground in people's attempts to construct more comprehensive unified theories (under the label Grand Unified Theory).
The obvious difficulty is that the straightforward and well-understood application of quantum mechanics to, say, the calculation of the behaviour of electrons in semiconductors cannot be presented to students as the routine calculus that it has become while the same students must know what uncertainties beset other parts of the subject.

One of the new generation of students, Joseph Godfrey from the University of Notre Dame, has now provided an interesting and stimulating pointer to the resolution of some of these questions (Phys. Rev. Lett. 52, 1365; 1984). Godfrey's contribution is refreshing not merely because he thanks his fellow graduate students for "discussions and support" but also because his article is, in its way, an important historical pointer. For the startingpoint is in the prehistory of quantum mechanics, the early (1927 and thereafter) contributions of Fritz London and Hermann Weyl to the argument about the meaning of the then new quantum theory. Godfrey's objective is to derive Dirac's commutator, the assumption that the operators representing conjugate dynamical variables in quantum theory anti-commute with each other in the sense that their order cannot be inverted without affecting the result, from London's paper on the subject (Z. Phys. 42, 375; 1927).

Godfrey remarks that it is "unfortunate" that the importance of London's paper has been ignored all these years. That, no doubt, is true but is also understandable. London in 1927 was attempting something that must already have seemed old-fashioned to many of his contemporaries - to account for Bohr's part-classical quantization rules from dynamical principles. Eventually, London's work allowed him to contribute significantly to the understanding of superconductivity, but even in the 1920 s it stimulated Weyl to generalize his earlier account of the projective geometry of the electromagnetic field to a consideration of vector fields in general and to a statement of the importance of gauge theory (as when, in electromagnetism, it is possible to multiply any solution for the vector potential of a field by complex factors without affecting the physically-observable forces).

Godfrey begins with Weyl's statement of what happens when a field vector is consistently displaced around a small circuit in relativistic space. Ordinarily, the result is to demonstrate both that Maxwell's equations are natural and, in non-Riemannian geometry such as that of general relativity, to obtain the link between electromagnetism and general relativity in which both matter and electromagnetism contribute to Einstein's energy-momentum tensor. Godfrey's innovation is to generalize Weyl's form, the simplest, of the non-Riemannian part of the result of the displacement of a vector around a small circuit in such a way as to mix together the position of a particle and the vector-potential which it experiences. This procedure is, to say the least, interesting; Godfrey points out that it is tantamount to assuming that the geometrical properties of space are determined in part by the character of the particle being used to probe that space, "but this is what is required to derive the Dirac commutator from the formalism".

The latest in what seems to be a spate of broodings about the fundamentals of quantum mechanics is a derivation of an axiom of Dirac's from Weyl's projective geometry.

The argument (unlike London's) has the virtue of treating the position and momentum of a particle on an equal footing. The notion that the operators by which they are represented should anti-commute in the sense that the difference of their products in one order and the other should be the imaginary number $i k$ (where $i$ is the square root of -1 and $h$ is Planck's constant divided by $2 \pi$ ) tumbles out. So too, Godfrey argues, does the exclusion principle. But by translating Weyl's geometric arguments to phase space, in which the position and momentum of a particle are treated similarly, Godfrey arrives at equations of motion for an electron even in special relativity which suggest ways in which the classical problem of the superficially infinite self-mass of a charged particle may be circumvented, as may be the corresponding renormalization procedures of Feymann and Schwinger in the case of quantum electrodynamics.

Godfrey's article is at this stage admittedly a sketch of a manuscript yet to be written (which is another of its refreshing qualities). His programme is ambitious, even for one still a graduate student. But if there turns out to be a castiron way of inferring the whole of quantum mechanics from some simple geometrical statements along the lines outlined by Weyl half a century ago, the consequences should be an end to the balkanization of the subject.
John Maddox 